\title{
Field Induced Structural Transition in Mesocrystallites
}

\author{
Weijia Wen, Ning Wang, Hongru Ma, Zhifang Lin, Wing Yim Tam, C. T. Chan, and Ping Sheng \\ Department of Physics, The Hong Kong University of Science and Technology, Clear Water Bay, Hong Kong, China
} (Received 11 December 1998)

We have fabricated multiply coated glass spheres with diameters of $45( \pm 2) \mu \mathrm{m}$ that exhibit appreciable magneto-, as well as electrorheological responses. Under crossed electric and magnetic fields, the microspheres were observed to form columnar crystallites with a structure that transforms from body-centered tetragonal to face-centered cubic as the ratio between the magnetic and the electric fields exceeded a minimum value. The observed transition scenario is in excellent agreement with calculations based on the model of field induced crystal formation and structural transition. [S0031-9007(99)09153-X]

PACS numbers: 64.70.Kb, 61.46.+w, 77.84.Lf

Crystals with lattice constants ranging from a few thousand angstroms to tens of microns - mesocrystals for short-constitute an interesting class of artificial materials with unique photonic properties. At present there are only a limited number of approaches for their realization, such as colloidal crystallization, 3D lithography, and the electrorheological (ER) or the magnetorheological (MR) effect. The ER or MR fluids are generally particle suspensions where the particles have large electric polarizability or magnetic permeability/magnetic moments. The ER or MR effect offers a unique technique whereby the suspended particles can be self-assembled into body-centeredtetragonal (bct) mesocrystallites [1-5] through the application of an external field. When ER and MR effects are combined, the EMR effect can lead to nonlinear increases in the yield stress, attributed to changes in the network structure [6]. In this work, we demonstrate for the first time that martensiticlike structural transformations can be realized under crossed electric $(E)$ and magnetic $(\mathrm{H})$ fields, when the $H / E$ ratio exceeds a minimum value. The observed transformation is correctly predicted by the minimization of combined electrostatic and magnetostatic free energy densities.

The field-controlled structural transformation is realized with multiply coated glass spheres with diameters of $45( \pm 2) \mu \mathrm{m}$ that exhibit significant EMR responses. Uniform glass spheres with diameters of $34 \pm 2 \mu \mathrm{m}$ were coated with $\mathrm{Ni}$ using the conventional electroless plating technique [7]. A Ni coating of approximately $2 \mu \mathrm{m}$ is desired. A layer of PZT (lead zirconate titanate) was coated on top of the Ni layer by using the sol-gel method [8] to prevent $\mathrm{Ni}$ oxidation in the subsequent annealing process, necessary for obtaining the desired magnetization. The PZT-coated spheres were heated in a vacuum chamber at $400{ }^{\circ} \mathrm{C}$ for 2 hours and then annealed at $550{ }^{\circ} \mathrm{C}$ for 3 hours. While not absolutely necessary, we have further coated another layer of $\mathrm{Ni}$, followed by a layer of $\mathrm{TiO}_{2}$, with processing steps similar to those described above and reported elsewhere [9]. The outer coatings were intended to give a large ER response [9]. The cross-sectional scanning electron microscope (SEM) pictures of the EMR spheres, together with the response to a small magnet, are shown in Fig. 1.

Experiments on EMR fluids were carried out by mixing the EMR spheres with silicon oil, and placing the sample in a cell with four electrodes. The high $E$ field, at $50 \mathrm{~Hz}$ and up to $2 \mathrm{kV} / \mathrm{mm}$, was applied across the top and bottom electrodes, separated $3 \mathrm{~mm}$ apart. Another two parallel

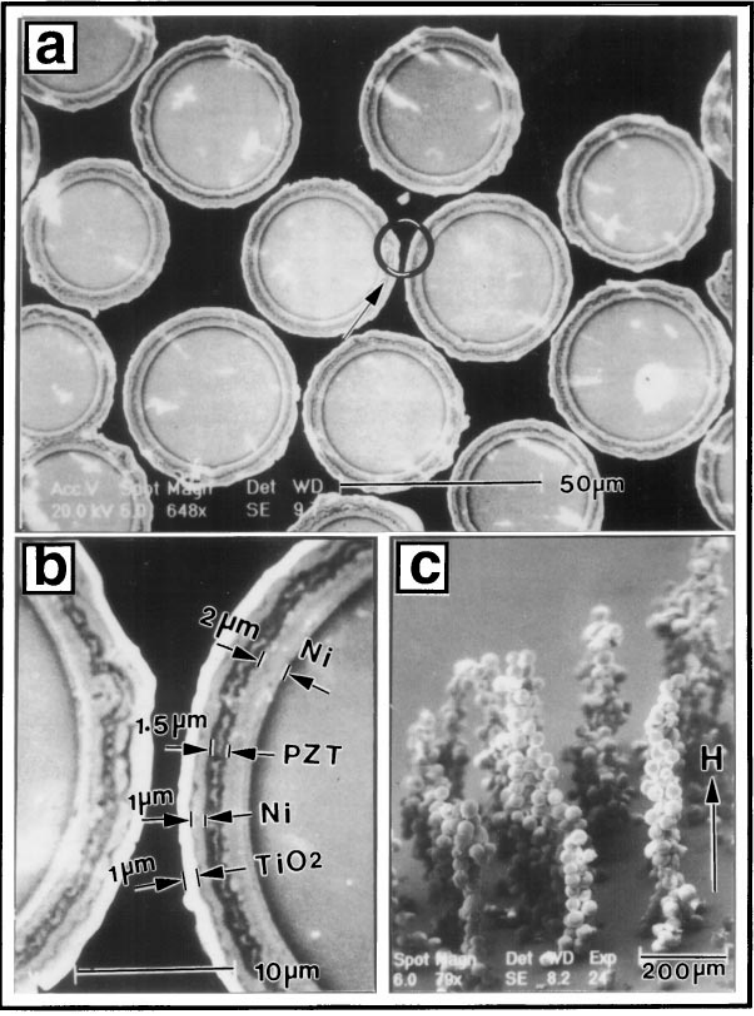

FIG. 1. (a) Cross-sectional SEM picture of the coated spheres. The apparent size variation is caused by deviation of the spheres' centers from the cutting plane. The arrow points to a circular region detailed in (b), which shows detailed thickness of the four coatings. From the inside out: $2 \mu \mathrm{m} \mathrm{Ni}$, $1.5 \mu \mathrm{m}$ PZT, $1 \mu \mathrm{m} \mathrm{Ni}$, and finally $1 \mu \mathrm{m} \mathrm{TiO}$. (c) The coated EMR spheres under the influence of a small magnetic stirrer. 
plates were mounted on two sides, $6 \mathrm{~mm}$ apart, and connected to a HP4282A LCR meter. The whole cell was placed in the central region of an electromagnet (GNW Magnet System, model 3470) with a pole surface diameter of $40 \mathrm{~mm}$ and a gap of $20 \mathrm{~mm}$. Without external fields, the suspension had the appearance of a random dispersion. At an $E$ field of 1 to $2 \mathrm{kV} / \mathrm{mm}$, columns appeared, with diameters typically ranging from seven to nine particles. At fixed $E$ field, the structural changes induced by the $H$ field inside the mesocrystallites were monitored by measuring the small dielectric constant changes in a direction perpendicular to both the $E$ and $H$ fields. The results are summarized in Fig. 2 for a sample with $20 \%$ solid volume fraction, at four values of the $E$ field. They have a characteristic dip on the order of $3 \%$, with the minimum position increasing linearly with increased $E$ field as shown in the upper-right inset. The slope of this variation gives the conversion factor between the strengths of ER and MR responses for our EMR spheres. The dielectric constant at the small $\mathrm{H}$-field region was flat and reversible. For the curve with $E=2 \mathrm{kV} / \mathrm{mm}$, this region occurred for $H<30 \mathrm{G}$. Irreversibility sets in at $H$ fields greater than this value. We obtained many cross-sectional micrographs by freezing in solid epoxy the configurations at various $H$-field values, and cutting the resulting samples. Four such micrograph pictures are shown in Fig. 3, with $E=2 \mathrm{kV} / \mathrm{mm}$. Figures 3(a) and 3(b) are for the configuration under zero $H$ field, cut along the (001) plane [Fig. 3(a)] and the (110) plane [Fig. 3(b)]. Taken together, they give direct evidence

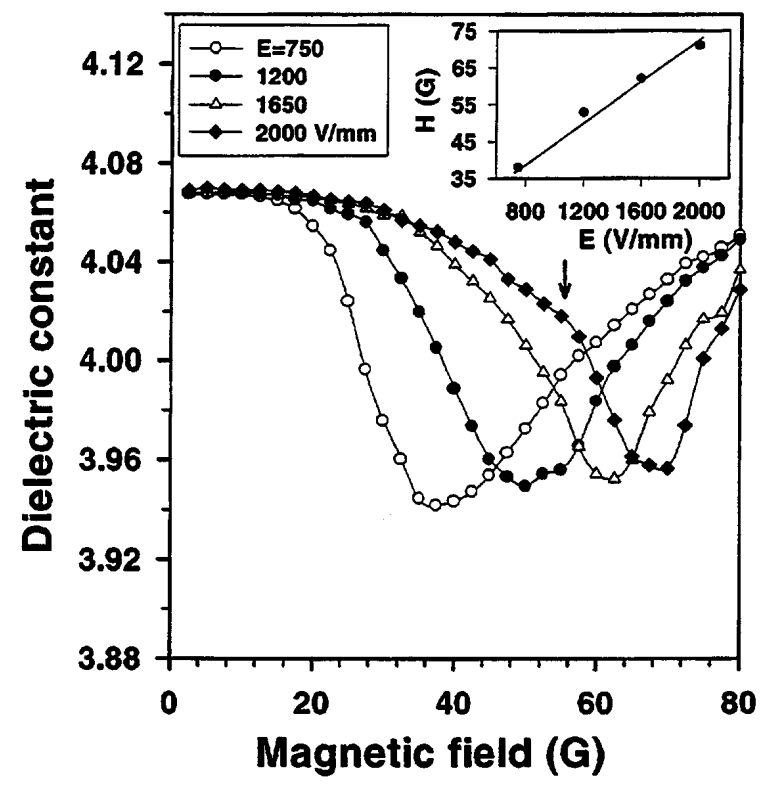

FIG. 2. The sample dielectric constant measured along the $y$ direction (perpendicular to both the $E$ and $H$ fields) as a function of the applied $H$ field, under four different $E$ fields. The upper right inset shows that the position of the minimum is a linear function of the applied $E$ field. The arrow indicates the $H$-field value where the fcc structure was observed. to the BCT structure. Figures 3(c), cut along the (011) plane, and 3(d), cut along the (110) plane, are for the configuration at $H=54 \mathrm{G}$. They clearly indicate a square lattice in the (011) plane (the fcc $\{100\}$ ) and a hexagonal lattice in the (110) plane (the fcc $\{111\}$ ), both the signatures of a fcc structure. Other cuttings were taken at 20,30, 35, 38, 40, and $50 \mathrm{G}$. Together with the dielectric constant measurements, they gave the following picture of the BCT-fcc transformation. At $H<30 \mathrm{G}$, only the BCT structure was seen. From 30 to $50 \mathrm{G}$, there was an apparent coexistence of local BCT structures with locally non-BCT structures. This coincides approximately with the onset of irreversibility in the dielectric constant measurement. At $54 \mathrm{G}$, where Figs. 3(c) and 3(d) were taken, only the fcc structure was seen. At $H>54 \mathrm{G}$, we see visually the columns being pulled apart and forming a "fractallike" structure at the minimum of the dielectric constant. Even higher $H$ fields result in the reformation of the columns aligned in the $H$-field direction, similar to the observations reported in Ref. [6]. We note here that the cohesive energy per particle (relative to random dispersion) in our system is at least 5 orders of magnitude larger than the thermal energy, and hence temperature effects are negligible. The transition between two structures of distinct symmetries was accomplished by the cooperative displacements of the spheres without any long range diffusion. As such, it can be regarded as a martensitic transformation. As the BCT and the fcc structures are close in energy under an $E$ field alone, the structural transition is induced by varying the relative strengths of the two external fields. However, as will be exposed later, the macroscopic columnar structure of these mesocrystallites plays a subtle but important role in deciding the fcc orientation. We emphasize that fcc becomes the ground state structure when electrostatic and magnetostatic energies

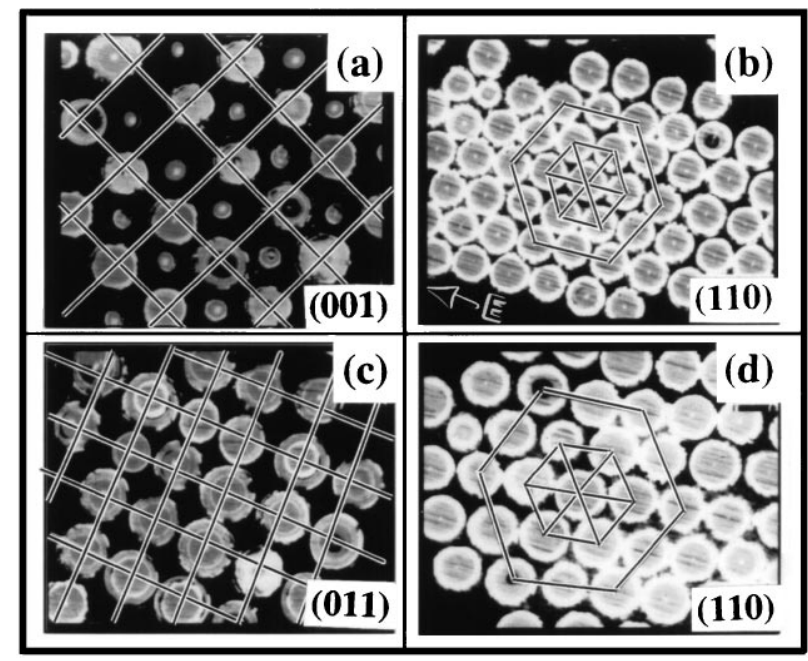

FIG. 3. Panels (a) and (b) are SEM cross-sectional pictures for a sample frozen (in epoxy) at $E=2 \mathrm{kV} / \mathrm{mm}$ with zero $H$ field, while (c) and (d) are cross sections for a sample frozen at $E=2 \mathrm{kV} / \mathrm{mm}$ and $H=54 \mathrm{G}$. See text for details. 
compete under crossed field conditions. In an applied magnetic field alone (with no electric field), the ground state microstructure is also BCT [10].

In our calculations, we assume that the spheres form columns with diameter ranging from 7 to 9 times the diameter of the spheres, and the preferred arrangement of the spheres inside the columns is determined through energy minimization. We consider the combined Gibbs free energy densities of the EMR fluid, $F=F_{E}+F_{H}$, where $F_{E}$ and $F_{H}$ are the electrostatic and magnetostatic parts of the free energy, respectively. $F$ is a function of the external fields, the arrangement of the spheres, as well as the dielectric and magnetic properties of the coated spheres and the fluid medium.

The electrostatic free energy density is given by $F_{E}=$ $-\bar{\varepsilon}_{z z} E^{2} / 8 \pi$. Here $z$ is the direction of the $E$ field $E$, maintained by a constant potential across two capacitor plates, and $\bar{\varepsilon}_{z z}$ is the $z z$ component of the effective dielectric tensor for the composite system. To simplify the calculations, we approximate the dielectric polarizability of the EMR spheres by that of the $\mathrm{TiO}_{2}$ coating (which is a lower bound due to the inner $\mathrm{Ni}$ coatings). The overall $\bar{\varepsilon}_{z z}$ can be found as $\bar{\varepsilon}_{z z}=f_{c} \varepsilon_{z z}+\left(1-f_{c}\right) \varepsilon_{2}$, where $f_{c}$ is the volume fraction of the mesocrystallite columns, fixed at the experimental value of $0.2 / p_{c}$, with $p_{c}$ the solid fraction in the mesocrystallites. The $\varepsilon_{z z}$ of the mesocrystallite, treated as a two-component composite, may be rigorously calculated for any given microstructure through the Bergman-Milton representation [5,11], with the input parameters of $\varepsilon_{2}=2.5$ for the dielectric constant of the fluid and $\varepsilon_{1}=85$ for that of $\mathrm{TiO}_{2}$ [12].

Each EMR sphere has a permanent moment $\vec{m}_{i}$ of approximately $1.5 \times 10^{-6} \mathrm{emu}$ in magnitude estimated from its $\mathrm{Ni}$ coating (assuming a magnetization of $58 \mathrm{emu} / \mathrm{g}$ ). The magnitude of this moment is assumed to be fixed, but its orientation can change depending on the arrangement of the spheres and the strength of the $H$ field, as determined by the minimization of the magnetostatic free energy density:

$$
F_{H}=F_{m}-\vec{M} \cdot \vec{H}+2 \pi \eta_{\alpha \beta} M_{\alpha} M_{\beta} .
$$

The first term,

$$
F_{m}=-\frac{1}{2 \Omega} \sum_{i} \vec{m}_{i} \cdot \sum_{j \neq i} \frac{1}{r_{i j}^{3}}\left[3 \hat{n}\left(\hat{n} \cdot \vec{m}_{j}\right)-\vec{m}_{j}\right],
$$

is the dipolar interaction due to the permanent moments. The second term is the interaction between the applied $H$ field and the magnetization $\vec{M}$ of the entire EMR system. The third term gives the depolarization effect, where $\eta_{\alpha \beta}$ are the demagnetization factors of the rectangular EMR cell (taken to be diagonal and $\eta_{x x}=\eta_{y y}=\frac{1}{4}$ and $\eta_{z z}=\frac{1}{2}$ ). To evaluate the $F_{H}$, we determine the orientation of each individual $\vec{m}_{i}$ through a spin dynamics simulation. A set of dynamical equations is derived from a Lagrangian $L=T-F_{H}$, where $T$ is an auxiliary kinetic energy that is dissipated slowly to allow the spins to evolve towards an energy minimum. We assume the cylindrical columns to be arranged in a square lattice. Each spin is allowed to vary freely, with the periodic boundary condition imposed in the $z$ direction. Our approach is noted to differ from the previous theoretical considerations $[2,13]$ in the following aspects: (i) the macroscopic columar structure is explicitly considered; (ii) the dielectric part of the interaction energy is computed with the Bergman-Milton formalism rather than the usual dipole approximation; (iii) permanent (rather than induced) magnetic moment is considered in better correspondence with the material properties; (iv) we do not assume uniform alignment of the magnetic moments, which is unphysical in our case. We note that if the magnetic moments were induced rather than permanent, a separate calculation [14] showed that the results are qualitatively the same.

The BCT, fcc, and the intermediate structures inside the mesocrystallite columns can all be described by a body-centered-orthorhombic unit cell with two spheres per cell. We align the $c$ axis with the $E$ field ( $z$ direction) and the $a$ axis with the $H$ field ( $x$ direction). We found that up to $H=60 \mathrm{G}$, the minimum energy state is associated with $c=2 R$ (i.e., touching spheres in the $E$ field direction). In addition, the spheres must be in physical contact with each other, as required by the fact that the compressional electrostatic and magnetostatic forces are stabilized by the steric repulsion of the spheres [15]. At $H=0$, the ground state is the BCT structure, with $a / c=\sqrt{6} / 2$ (and $b=a$ ), as seen experimentally and in agreement with prior works [1-4]. The (110) plane is a closed-packed plane and remains invariant under the $\mathrm{BCT} \rightarrow$ fcc transformation. Together with the constraint of $c=2 R$, the transformation path can be described by the equation $b^{2}=12 R^{2}-a^{2}$. The hard sphere condition $(a \geq 2 R, b \geq 2 R)$ requires $1 \leq a / c \leq \sqrt{2}$, with the limits corresponding to fcc structures oriented differently with respect to the $H$ field. We performed energy calculations along this path, and the results are shown in Fig. 4 for different values of $H$-field strengths with a fixed applied $E$ field of $2 \mathrm{kV} / \mathrm{mm}$. We find good quantitative agreement with the experiments by using $\left|\vec{m}_{i}\right|=1.0 \times 10^{-6} \mathrm{emu}$. Thus, the only parameter used in the calculation agrees well with the value estimated from the Ni coating thickness.

From Fig. 4, we see that for $H$ fields below $\sim 30 \mathrm{G}$, $\mathrm{BCT}$ remains the optimal structure. This agrees with our observation that there is a reversible regime in the dielectric constant measurement for fields somewhat below $30 \mathrm{G}$. For $H$ fields above $30 \mathrm{G}$, the minimum free energy state rapidly moves to the fcc structure. This coincides with the observation of non-BCT structures at these $H$ fields; the observed BCT structure could be due to defects and the lack of temperature effect to overcome local metastabilities. At $57 \mathrm{G}$, fcc is a stable minimum free energy state, as compared to the observation of the clean fcc state at $54 \mathrm{G}$. 


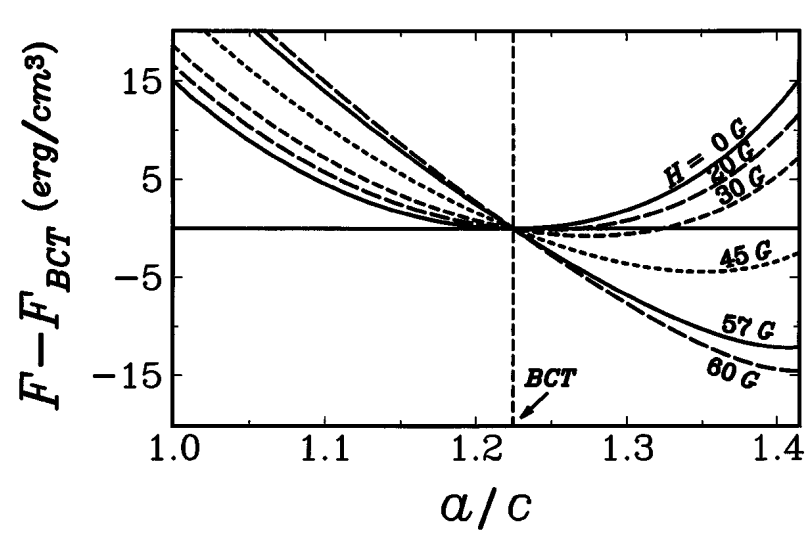

FIG. 4. Calculated sum of the electrostatic and magnetostatic free energy densities, minimized with respect to the $c$ and $b$ axes values, are plotted as a function of the $a / c$ ratio. The energy of the BCT structure is used as reference.

We note that the columnar structure breaks the cubic symmetry of the fcc structure with $a / c=1$ or $\sqrt{2}$, because the demagnetization effect favors the deformation where $a / c$ increases.

The fact that $a$ increases under the action of the $H$ fields implies a decrease of $\bar{\varepsilon}_{y y}$. This is indeed observed as shown in Fig. 2. Based on effective medium theory, we estimate that $\bar{\varepsilon}_{y y}$ should decrease by about $0.3 \%$ to $5.5 \%$ (depending on the shape of the columns and their orientation) as the internal structure changes from BCT to fcc. The observed decrease of about $1 \%$ falls within this range.

The excellent consistency achieved between theory, experiment, and characterization means that the observed structural transformation may be characterized as an external-field-induced martensiticlike transformation. The recognition of this mechanism opens a new avenue for the fabrication of mesoscrystals with BCT and fcc structures (and those in between), with unique and tunable optical characteristics.

We thank Dr. X.X. Zhang for discussions. We acknowledge support by HKUST-RI 93/94.SC09, CERG HKUST-6142/97P and 6136/97P, and the William Mong Solid State Cluster Laboratory.

[1] R. Tao and J. M. Sun, Phys. Rev. Lett. 67, 398 (1991); Phys. Rev. A 44, R6181 (1991).
[2] G. Bossis, H. Clercx, Y. Grasselli, and E. Lemaice, in Electrorheological Fluids, edited by R. Tao and G. D. Roy (World Scientific, Singapore, 1994), p. 153; H. Clercx and G. Bossis, Phys. Rev. E 48, 2721 (1993).

[3] L. C. Davis, Phys. Rev. A 46, R719 (1992).

[4] R. Friedberg and Y. K. Yu, Phys. Rev. B 46, 6582 (1992).

[5] H. Ma, W. Wen, W. Y. Tam and P. Sheng, Phys. Rev. Lett. 77, 2499 (1996).

[6] K. Koyama, in Electro-Rheological Fluids and MagnetoRheological Suspensions and Associated Technology, edited by W. A. Bulloguch (World Scientific, Singapore, 1996), p. 245.

[7] J. W. Severin, R. Hokke, H. Venderwel, and G. deWith, J. Electrochem. Soc. 140, 682 (1983).

[8] C. J. Briker and G.W. Scherer, Sol-Gel Science: The Physics and Chemistry of Sol-Gel Processing (Academic Press, New York, 1990).

[9] W. Y. Tam, G. H. Yi, W. Wen, H. Ma, M. M. T. Loy, and P. Sheng, Phys. Rev. Lett. 78, 2987 (1997).

[10] L. Zou, W. Wen, and P. Sheng, Phys. Rev. Lett. 81, 1509 (1998).

[11] D. J. Bergman, Phys. Rep. 43, 377 (1978); Phys. Rev. B 19, 2359 (1979); G. W. Milton Appl. Phys. A 26, 1207 (1981); J. Appl. Phys. 52, 5286 (1980).

[12] The dielectric constant of $\mathrm{TiO}_{2}$, depending on its crystalline orientation with respect to the applied $E$ field, is 85 or 170 . Here, for lack of information supporting a highly crystalline state, we use the lower value.

[13] See, e.g., T. Tao, Phys. Rev. E 57, 5761 (1998).

[14] If the magnetic moments were induced, the magnetostatic free energy can be written as $-\mu_{x x} H^{2} / 8 \pi$. Here $\mu_{x x}$ is the component of the effective magnetic permeability tensor along the direction of the $H$ field, which can be calculated with the Bergman-Milton formalism on exactly the same footing as the electrostatic part of the free energy. We have chosen to model our system as a system of permanent magnetic moments because that is closer to reality. The coated spheres possess permanent moments, and a separate measurement showed that the induced moment is only a fraction of the permanent moment up to $H=60 \mathrm{G}$.

[15] If we allow the spheres to come apart, there are multiple deformation paths that can bring the columnar mesocrystallites into other arrangements that can have lower energy than the fcc structure (such as very oblate columns with hexagonal-close-packed internal structure). However, the transformation barrier involved is orders of magnitude higher than the thermal energy, and such paths have no physical relevance. 